\title{
Sessão de Tai Chi Chuan promove hipotensão pós-exercício e redução da sobrecarga cardiovascular em idosos hipertensos
}

\author{
Tai Chi Chuan session promotes post-exercise hypotension and reduces cardiac \\ overload in elderly hypertensive patients
}

Raphael Santos Teodoro de Carvalho ${ }^{1}$, Anderson Gregorio Joaquim², Joab Jefferson da Silva Xavier ${ }^{3}$, Vivian Marques Miguel Suen ${ }^{4}$, Leila Maria Marchi Alves Ancheschi ${ }^{5}$

\begin{abstract}
RESUMO
Modelo: Estudo intervencional, prospectivo e quantitativo. Objetivo: Analisar o comportamento da pressão arterial (PA) e duplo produto (DP) de idosos hipertensos, após uma sessão de Tai Chi Chuan (TCC). Métodos: Participaram do estudo 20 idosos hipertensos em que as variáveis hemodinâmicas foram medidas pré-sessão de TCC e sessão controle (sem exercício), e monitorada durante 60 minutos após término da sessão de TCC e controle. Foi respeitado um intervalo de 7 dias entre uma sessão e outra. Resultados: Após término da sessão de TCC, a frequência cardíaca permaneceu elevada por 30 minutos retornado aos valores pré-exercício a partir do $40^{\circ}$ minuto. Observou-se redução significativa da pressão arterial média (PAM) a partir do $10^{\circ}$ minuto, e redução significativa da pressão arterial sistólica (PAS) e diastólica (PAD) a partir do $20^{\circ}$ minuto até o $60^{\circ}$ minuto após sessão de TCC $(p<0,001)$. Houve redução em relação aos valores pré-exercício de aproximadamente $-22 /-18 \mathrm{mmHg}$ para PAS e PAD respectivamente $(p<0,001)$. Observou-se redução significativa do duplo produto (DP) a partir do $30^{\circ}$ minuto, continuando a reduzir até o $60^{\circ}$ minuto após TCC $(p<0,001)$. A magnitude de redução do DP comparado ao momento pré-exercício foi na ordem de $1675(p<0,001)$. Conclusão: Esses achados sugerem que única sessão de TCC promove hipotensão pós-exercício (HPE) e reduz a sobrecarga cardíaca em idosos com HAS.
\end{abstract}

Palavras-chaves: Hipotensão Pós-Exercício. Hipertensão. Tai Chi Chuan. Idosos.

\begin{abstract}
Design: Interventional study, prospective and quantitative study. Objective: To determine blood pressure and double product values of elderly hypertensive patients, after a single Tai Chi Chuan (TCC) session. Methods: Twenty elderly hypertensive patients participated in the study, in which the hemody-
\end{abstract}

1. Doutorando em ciências pela Escola de Enfermagem de Ribeirão Preto da Universidade de São Paulo (EERP-USP), Ribeirão Preto, SP, Brasil

2. Mestrando em ciências pelo Departamento de Clínica Médica da Faculdade de Medicina de Ribeirão Preto da Universidade de São Paulo (FMRP-USP),, Ribeirão Preto, SP, Brasil

3. Doutor em ciências pela EERP-USP.

4. Professora doutora do Departamento de Clínica Médica da FMRP-USP.

5. Professora doutora associada Do Departamento de Enfermagem Geral e Especializada da EERP-USP.
CORRESPONDÊNCIA:

Raphael Santos Teodoro de Carvalho Universidade de São Paulo, Departamento de Enfermagem Geral e Especializada da Escola de Enfermagem de Ribeirão Preto. Avenida dos Bandeirantes, 3900. CEP: 14040-902, Ribeirão Preto - SP, Brasil. 
namic variables were measured pre-TCC session and control session (without exercise), and monitored for 60 minutes after the end of both sessions. Each session had an interval of 7 days. Results: The results showed that at the end of the TCC, the heart rate remained elevated for 30 minutes, returning to pre-exercise values from the $40^{\text {th }}$ minute. Significant reduction in mean blood pressure (MBP) from the 10th minute, and significant reduction in systolic (SBP) and diastolic (DBP) blood pressure from the $20^{\text {th }}$ minute to the $60^{\text {th }}$ minute after TCC session $(p<0.001)$ were observed. A magnitude reduction compared to pre-exercise was around $-22 /-18 \mathrm{mmHg}$ for SBP and DBP respectively $(\mathrm{p}<0.001)$. Significant reduction of the DP was observed from the 30th minute, and reduced until the 60th minute after TCC $(p<0.001)$. The magnitude of DP reduction compared to pre-exercise was around $-1675(p<0.01)$. Conclusions: These findings show that single TCC session promotes post-exercise hypotension (PEH) and reduces cardiac overload in elderly hypertensive patients.

Keywords: Post-Exercise Hypotension. Hypertension. Tai Chi Chuan. Elderly.

\section{Introdução}

Observamos um crescente aumento da população idosa ao longo dos anos. Segundo relatório do Banco Mundial, ${ }^{1}$ no ano de 2010 a população brasileira de idosos acima de 65 anos era próximo de 18 milhões, o que representava cerca de $12 \%$ da população. Estima-se que em 2050, haverá cerca de 65 milhões de idosos no Brasil, totalizando aproximadamente $49 \%$ da população brasileira. Logo, aumenta-se a preocupação dos profissionais, principalmente os da área da saúde, pois com o avanço da idade, a deterioração estrutural e funcional ocorre na maioria dos sistemas fisiológicos. Essas mudanças relacionadas à idade afetam diversos tecidos e órgãos, o que compromete a realização das atividades da vida diária e qualidade de vida dos idosos. ${ }^{2,3}$

O processo de envelhecimento é agravado com o sedentarismo, propiciando alterações na composição corporal como o aumento da gordura corporal, principalmente na região abdominal e a perda de massa muscular. Esses fatores aumentam o risco de desenvolvimento de doenças metabólicas e cardiovasculares tais como, a obesidade, diabetes tipo 2, doença arterial coronariana, dislipidemia e hipertensão arterial sistêmica (HAS), aumentando a chance de morte dessas pessoas. ${ }^{4-10}$

Uma dessas doenças que necessita de abordagem multidisciplinar para a prevenção e tratamento é a HAS. Segundo a Sociedade Brasileira de Cardiologia, ${ }^{11}$ a HAS é uma condição clínica que envolve vários fatores, sendo caracterizada pelos níveis sustentados de pressão arterial sistólica (PAS) e diastólica (PAD) acima de 140 e/ou 90 mmHg res- pectivamente. No Brasil, a prevalência de HAS nos idosos é de $52,7 \%$ entre os indivíduos de 65 a 74 anos, e 55\% entre os indivíduos acima de 75 anos de idade. Nos últimos anos estima-se que as complicações advindas da HAS, geraram prejuízos no trabalho e na renda das famílias de aproximadamente 4,18 bilhões de reais. Além disso, a HAS é responsável por aproximadamente $45 \%$ das mortes por doença cardíaca e $51 \%$ das mortes advinda do acidente vascular cerebral (AVC). ${ }^{11}$

Tem sido proposto por meio de revisões científicas, que mudanças no estilo de vida, tais como: menor consumo de álcool, tabaco e sódio, aumento da ingestão de potássio e controle do peso corporal, atuam como intervenções não medicamentosas na prevenção e tratamento da HAS. ${ }^{11,12,13} \mathrm{E}$ ultimamente, tem ganhado destaque a recomendação para realização de exercício físico, a fim buscar o controle pressórico principalmente no estado de repouso, propiciando menor estresse cardíaco. ${ }^{11,13,14}$ Diversos estudos têm demonstrado que a realização de uma única sessão de exercício físico promove redução da PA a valores inferiores aos medidos antes do exercício. A este fenômeno foi atribuído o nome de hipotensão pós-exercício (HPE). ${ }^{14-17}$

As crescentes pesquisas sobre HPE indicam que os exercícios aeróbios que recrutam grande massa muscular, como os exercícios de caminhada, corrida e exercício em bicicleta, apresentam maior HPE em relação aos exercícios resistidos. ${ }^{14,15,16,18}$ Apesar dos estudos ${ }^{14-18}$ mostrarem que ocorre HPE em exercícios aeróbios, são escassos os trabalhos que analisaram a HPE em outros tipos de exercícios físicos praticados pela população idosa, como o Tai Chi Chuan (TCC). ${ }^{19}$ 
O TCC originário da China é um exercício que se caracteriza por ter movimentos individuais sincronizados de um modo suave e contínuo. Os movimentos são integrados por meio de respiração profunda e concentração mental, o praticante pode adquirir um estado de harmonia entre corpo e mente, e o esforço para realização das aulas possuem característica de intensidade moderada. ${ }^{20} \mathrm{~A}$ literatura demonstra inúmeros benefícios aos praticantes do TCC, dentre eles: melhora da função físi$\mathrm{ca}^{21,22}$ melhora psicossocial, ${ }^{20,23}$ melhora da aptidão cardiorrespiratória ${ }^{24,25,26}$ e redução da pressão arterial. 27,28

Em relação ao tratamento da HAS, principalmente em idosos, pouco se conhece a respeito do comportamento de variáveis cardiovasculares e hemodinâmicas como a PAS, PAD, pressão arterial média (PAM), frequência cardíaca ( $F C$ ) e duplo produto (DP) frente à prática do TCC, mesmo sendo uma modalidade de exercícios com alta participação da população de idosos. ${ }^{22}$ Sendo assim, existe ainda uma lacuna na literatura a respeito de diferentes modalidades de exercícios, como o TCC, e o seu efeito sobre o tratamento da HAS. Acreditamos que a análise e interpretação das variáveis cardiovasculares e hemodinâmicas frente ao TCC possibilitará ampliar a visão sobre as diferentes práticas de exercícios e os seus benefícios para o tratamento não-medicamentoso da HAS. Logo, nosso objetivo foi analisar o efeito de uma única sessão de TCC sobre o comportamento da PAS, PAD, PAM, FC e DP em idosos com HAS.

\section{Materiais e Métodos}

Trata-se de um estudo intervencional, prospectivo e do tipo quantitativo. A amostra foi composta 20 idosos hipertensos (13 mulheres) com 30,9 $\pm 2,2 \mathrm{~kg} / \mathrm{m}^{2}$ de índice de massa corporal (IMC) que participavam de atividades assistenciais promovidas por uma entidade civil sem fins lucrativos de caráter filantrópico, localizada em um município do interior paulista. Todos os idosos se encontravam em terapia medicamentosa para controle da doença e não houve alteração durante o estudo.

A participação no estudo foi de forma voluntária e todos os idosos foram informados do objetivo do estudo, dos procedimentos a serem realizados, dos possíveis desconfortos, e assim, foram convidados a assinar o termo de consentimento livre e esclarecido. O presente estudo foi aprovado pelo Comitê de Ética em Pesquisa com Seres Humanos da Escola de Enfermagem de Ribeirão Preto da Universidade de São Paulo.

Foram incluídos no presente estudo idosos sedentários, isto é, que acumulavam menos de 20 minutos por dia ou menos que 150 minutos por semana de exercício físico controlado e planejado nos últimos 3 meses, ${ }^{29}$ que tinham liberação médica para prática de exercícios físicos, com diagnóstico de HAS estabelecida há pelo menos um ano e em terapia medicamentosa inalterada há pelo menos três meses. Foram excluídos idosos com alterações cardiovasculares, renais, pulmonares e doenças psicológicas que impossibilitasse a prática de exercícios físicos e valores de PA de repouso superiores a $160 / 100 \mathrm{mmHg}$ no momento que antecedia a prática do TCC.

Para avaliação antropométrica da estatura foi utilizado um estadiômetro (Filizola $\AA /$ Brasil) com resolução de um centímetro, a cabeça da pessoa foi posicionada com plano de Frankfürt (parte inferior da órbita ocular no mesmo plano do orifício externo do ouvido), sendo colocada a haste da régua no ponto vértex, ou ponto mais alto da cabeça.

A massa corporal foi medida em uma balança microeletrônica portátil (Filizola $囚 /$ Brasil), com precisão de $50 \mathrm{~g}$, capacidade de $200 \mathrm{~kg}$. Os voluntários foram posicionados de pé e de costas no centro da plataforma do aparelho, descalços e usando roupas leves, com o corpo o mais alongado possível. O IMC foi calculado considerando o peso e a estatura, sendo obtido por meio da seguinte equação: IMC = peso $(\mathrm{kg}) /$ estatura $^{2}(\mathrm{~m})$.

A circunferência abdominal foi obtida na menor curvatura localizada entre o rebordo costal inferior e a crista ilíaca, com fita métrica flexível e inelástica de precisão de $0,1 \mathrm{~cm}$, sem comprimir os tecidos. Quando não foi possível identificar a menor curvatura, obtivemos a medida dois centímetros acima da cicatriz umbilical. Todas as coletas seguiram os procedimentos sugeridos por Heyward. ${ }^{30}$

O protocolo experimental ocorreu de acordo com algumas visitas. Na primeira visita, foram coletadas as variáveis antropométricas e as variáveis hemodinâmicas no estado de repouso. Na segunda visita, os idosos foram submetidos a uma aula de TCC de intensidade moderada. Segundo o American 
College of Sports Medicine ${ }^{2}$ exercício físico aeróbio de moderada intensidade é definido como esforços executados entre 3 e 5,9 equivalentes metabólicos da tarefa, da sigla em inglês MET (Metabolic Equivalent Task). ${ }^{2}$ A sessão de TCC teve a duração de 60 minutos, sendo os 10 minutos iniciais de aquecimento, 40 minutos subsequentes composto por exercícios específicos de TCC e os 10 minutos finais de relaxamento. A sessão foi ministrada por um profissional de Educação Física habilitado para condução da aula. Após a sessão de TCC, foram coletadas por $60 \mathrm{mi}-$ nutos as variáveis fisiológicas FC, PAS e PAD.

A sessão controle foi realizada sete dias após a sessão em que foi realizado a prática de TCC. Nesta terceira visita, o grupo controle (não praticaram o TCC) composto pelos mesmos voluntários submetidos à aula de TCC. Foi respeitado o mesmo local e horário que ocorreu a sessão prática do TCC, porém, na sessão controle os voluntários permaneciam sentados por 60 minutos, e após esse tempo, coletava-se por 60 minutos as variáveis fisiológicas FC, PAS e PAD, similar à sessão de TCC. Ambas as sessões e a coleta das variáveis ocorreram entre 7:00 e 10:00 horas da manhã.

O monitoramento da pressão arterial e frequência cardíaca foram realizados pelo método indireto e oscilométrico, tanto no momento que antecedia a sessão de TCC e a sessão controle, quanto no monitoramento após as sessões, sendo utilizado os equipamentos modelo OMRON HEM 705 $\mathrm{CP} \circledast$, com braçadeiras adequadas a medida da circunferência braquial do paciente. Todas as exigências técnicas para adequada mensuração da PA antes e após o exercício físico foram de acordo com as especificações das VII Diretriz brasileira de hipertensão arterial. ${ }^{11}$ A PAS e a PAD foram medidas présessão de TCC e pré-sessão controle por três pesquisadores experientes após os indivíduos permanecerem sentados por 10 minutos. As medidas continuaram após a sessão de TCC e após a sessão controle por um tempo total de 60 minutos, sendo a medida realizada a cada 10 minutos com os indivíduos permanecendo na posição sentada durante todo o período de monitoramento. As medidas de FC seguiu a mesma ordem temporal das medidas de PA, em que para seu monitoramento foram utilizados cardiofrequencímetros modelo Pollar ${ }^{\circledR}$ FT6.

Dado que a PAM determina indiretamente a velocidade do fluxo sanguíneo para toda circulação sistêmica, seus valores foram obtidos a partir da seguinte equação ${ }^{31}: P A M=(2(P A D)+P A S) / 3$. Já o duplo produto que prediz indiretamente o trabalho do miocárdio frente à captação de oxigênio e o fluxo sanguíneo coronariano, seus valores foram obtidos a partir da equação ${ }^{32}$ : DP= PAS x FC.

Os resultados estão apresentados como média e desvio-padrão (desv-pad). Para normalidade na distribuição dos dados foi utilizado o teste de Shapiro-Wilk e a homogeneidade foi identificada em todas as análises pelo teste de Levene. A esfericidade dos dados foi verificada por meio do teste de Mauchly seguida pela correção de GreenhouseGeisser quando os pressupostos violados. Para análises das respostas da FC, PAS, PAD, PAM e DP após cada condição (em repouso e na sessão de TCC), foi utilizada análise de variância (ANOVA) para medidas repetidas, seguidas pelo teste post-hoc de Bonferroni. Adicionalmente, para diferença (delta) entre variáveis hemodinâmicas (pós-teste $60 \mathrm{mi}$ nutos - pré-teste), foi utilizado o teste t para amostras em pares. Os dados foram analisados através do pacote estatístico SPSS $\AA$ versão 21.0 , sendo atribuído um nível de significância estatística de 5\% para todas as análises.

\section{Resultados}

Na tabela 1 estão apresentadas a estatística descritiva das características gerais e variáveis hemodinâmicas observadas.

A Figura 1 (A) apresenta o comportamento da frequência cardíaca após as sessões experimentais. Observa-se que a FC permaneceu elevada até o $30^{\circ}$ minuto após sessão de TCC, não apresentando mudanças até o final do monitoramento $(p=0,68)$. Também não houve alterações da FC após sessão controle $(p>0,05)$. A Figura 1 (B) ilustra o delta de variação da FC após sessão controle $(-0,6 \pm 4,1$ bpm) e após TCC ( $0,3 \pm 4,9$ bpm), mostrando não haver diferenças significativas entre as duas condições $(p=0,51)$.

O comportamento da PAS e PAD após as sessões experimentais estão apresentadas na Figura 2 ( $A$ e $C$ ). Houve redução significativa nos valores de PAS após TCC $(p<0,05)$. Encontrou-se um efeito do tempo $(F(3,3 ; 62,2)=83,11 ; p<0,001)$, efeito da condição $(F(1 ; 19)=233,3 ; p<0,001)$ e interação condição e tempo $(F(3,5 ; 66,9)=212,15$; 
Tabela 1: Características dos participantes $(n=20)$

\begin{tabular}{|c|c|c|c|c|c|c|}
\hline Variáveis & Média & \pm & desvio-padrão & \multicolumn{3}{|c|}{ IC $(95 \%) *$} \\
\hline Idade (anos) & 70,8 & \pm & 4,4 & 68,7 & - & 72,8 \\
\hline Peso $(\mathrm{kg})$ & 80,3 & \pm & 4,8 & 78,02 & - & 82,5 \\
\hline Estatura $(\mathrm{cm})$ & 161,3 & \pm & 4,3 & 159,3 & - & 163,2 \\
\hline $\operatorname{IMC}\left(\mathrm{kg} / \mathrm{m}^{2}\right)$ & 30,9 & \pm & 2,2 & 29,9 & - & 31,9 \\
\hline Circunferência Abdominal $(\mathrm{cm})$ & 97,2 & \pm & 4,9 & 94,9 & - & 99,4 \\
\hline $\mathrm{FC}$ em repouso (bpm) & 77,2 & \pm & 3,7 & 75,5 & - & 78,9 \\
\hline PAS em repouso $(\mathrm{mmHg})$ & 149,1 & \pm & 2,8 & 147,8 & - & 150,4 \\
\hline PAD em repouso $(\mathrm{mmHg})$ & 92,6 & \pm & 2,8 & 91,3 & - & 93,9 \\
\hline PAM em repouso $(\mathrm{mmHg})$ & 111,4 & \pm & 2,1 & 110,4 & - & 112,4 \\
\hline DP em repouso $(\mathrm{mmHg} \times \mathrm{bpm})$ & 11507 & \pm & 530 & 11259 & - & 11755 \\
\hline
\end{tabular}

*IC95\% (Intervalo de Confiança de 95\%).
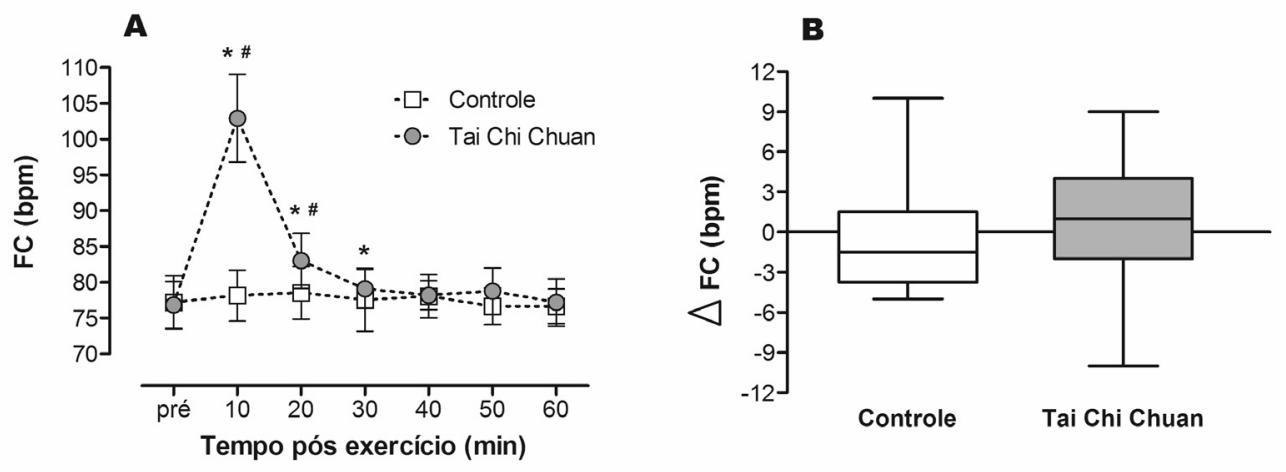

Figura 1. (Painel A) Comportamento da frequência cardíaca (FC) na sessão controle e pós sessão de Tai Chi Chuan. (Painel B) Delta “ entre os valores de FC após 60 minutos e pré sessões controle e Tai Chi Chuan. *diferente de pré sessão de Tai Chi Chuan; \# diferente da situação controle; para ANOVA (post-hoc Bonferroni, significância $p<0,05$ ). Dados apresentados como média \pm desv-pad, $n=20$.

$p<0,001)$. A mesma redução significativa foi encontrada para os valores de PAD $(p<0,05)$, sendo um efeito do tempo $(F(3,1 ; 59,2)=90,04 ; p<$ $0,001)$, efeito da condição $(F(1 ; 19)=653,6 ; p<$ $0,001)$ e interação condição e tempo $(F(3,6 ; 67,8)$ $=94,5 ; p<0,001)$.

Em relação ao momento pré-exercício $(152,2$ $\pm 3,8 / 91 \pm 3,5 \mathrm{mmHg}$ ), observou-se efeito hipotensor para PAS e PAD a partir do $20^{\circ}$ minuto após TCC $(142,2 \pm 4,3 / 85,5 \pm 3,1 \mathrm{mmHg}$ respectivamente), $p<0,001$. Esse mesmo efeito hipotensivo tanto para a PAS e PAD, não foi observado após sessão controle $(p>0,05)$. Já a Figura 2 ( $B$ e $D)$ ilustra o delta de variação da PAS e PAD após sessão controle $(0,1 \pm 4,5 /-0,8 \pm 4,1 \mathrm{mmHg})$ e após TCC $(-22,4 \pm 4,5 /-17,9 \pm 4,2 \mathrm{mmHg}$ respectivamente), mostrando significância na promoção da HPE apenas quando os participantes realizaram a sessão de TCC $(p<0,001)$.

O comportamento da PAM e DP após as sessões experimentais estão apresentadas na Figura 3 ( $A$ e $C)$. Houve redução significativa nos valores de PAM após o TCC $(p=0,01)$. Encontrou-se um efeito do tempo $(F(3,5 ; 82)=157,04 ; p<0,001)$, efeito da condição $(F(1 ; 19)=1099,85 ; p<0,001)$ e interação condição e tempo $(F(3,7 ; 70,4)=198,88$; $p<0,001)$. Para os valores de DP também foi observada redução significativa após TCC $(p=0,002)$, 

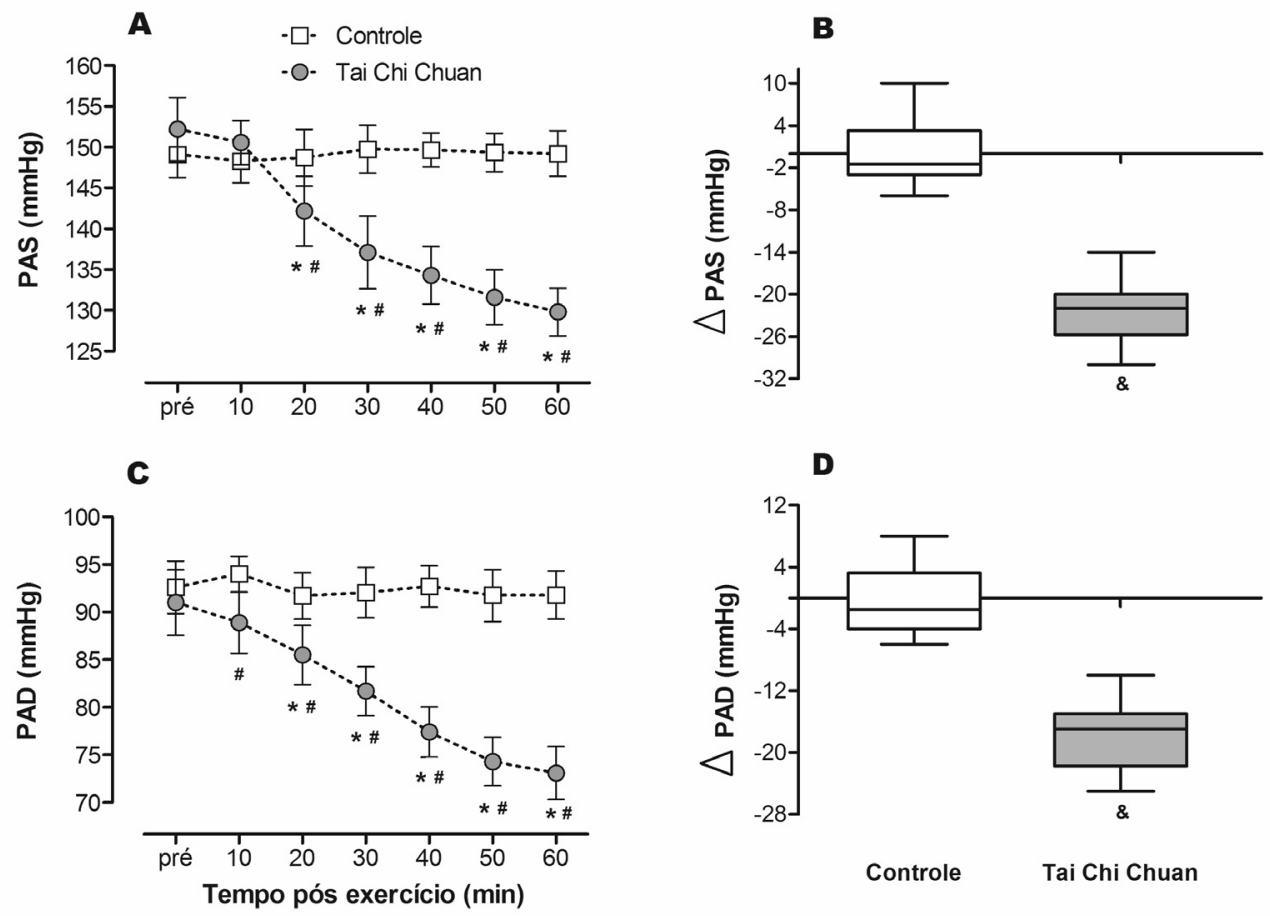

Figura 2. (Painel A e C) Comportamento da pressão arterial sistólica (PAS) e pressão arterial diastólica (PAD) respectivamente, nas sessões controle e após Tai Chi Chuan. (Painel B e D) Delta "entre os valores de PAS e PAD respectivamente, após 60 minutos e pré sessões controle e Tai Chi Chuan. *diferente de pré sessão de Tai Chi Chuan; \# diferente do controle; para ANOVA (post-hoc Bonferroni, significância $p<0,05)$. \& diferente do controle; teste T para amostras em pares $(p<0,05)$. Dados apresentados como média \pm desv-pad, $\mathrm{n}=20$.

sendo um efeito do tempo $(F(3,7 ; 69,6)=113,02$; $\mathrm{p}<0,001)$, efeito da interação condição e tempo $(F(3,4 ; 64,7)=160,2 ; p<0,001)$, sem qualquer efeito observado com relação condição, $p=0,76$. Não houve mudanças significativas na PAM e DP após a sessão controle $(p=0,23)$.

Em relação ao momento pré-exercício $(111,4$ $\pm 2,8 \mathrm{mmHg}$ ), observou-se redução significativa da PAM a partir do $10^{\circ}$ minuto após TCC $(109,5 \pm 2,5$ $\mathrm{mmHg}), p=0,026$. Já o DP após sessão de TCC, apresentou redução significativa partir do $30^{\circ} \mathrm{mi}-$ nuto em relação ao momento pré-exercício $(11694,1$ $\pm 524,2$ vs $10846,6 \pm 556,9$ ), sendo evidenciado redução da sobrecarga cardíaca apenas quando os participantes realizaram o exercício físico $(p<$ 0,001).

A figura 3 (B) apresenta o delta de variação da PAM, que quando comparado a sessão controle observamos significante redução da PAM apenas na situação em que os participantes realizaram o TCC $(-0,5 \pm 3,1 \mathrm{mmHg}$ vs $-19,4 \pm 3,3 \mathrm{mmHg}), \mathrm{p}<0,001$. Já na figura 3 (D) o delta de variação do DP após
TCC foi superior quando comparado a sessão controle $(-1674,8 \pm 707,7$ vs $-73,5 \pm 619,6)$, sendo essas condições significativamente diferentes $(p<$ 0,001).

\section{Discussão}

O objetivo principal do presente estudo foi analisar o efeito de uma sessão de TCC sobre o comportamento da PAS, PAD, PAM, FC e DP em idosos hipertensos, em que os resultados mostraram HPE significativa em relação aos valores pré-sessão de TCC e comparados à sessão controle, além da redução da sobrecarga cardíaca avaliada pelo menor DP. A HPE ocorreu tanto para a PAS quanto para a PAD a partir do $20^{\circ}$ minuto, ocorrendo mais reduções até o $60^{\circ}$ minuto pós-sessão. Além disso, a ausência de modificações na PA durante a sessão controle (sem a prática do TCC), confirmam que a diminuição observada foi dependente da realização da sessão de TCC e não das variações pressóricas diurnas normais e da terapia medicamentosa para 

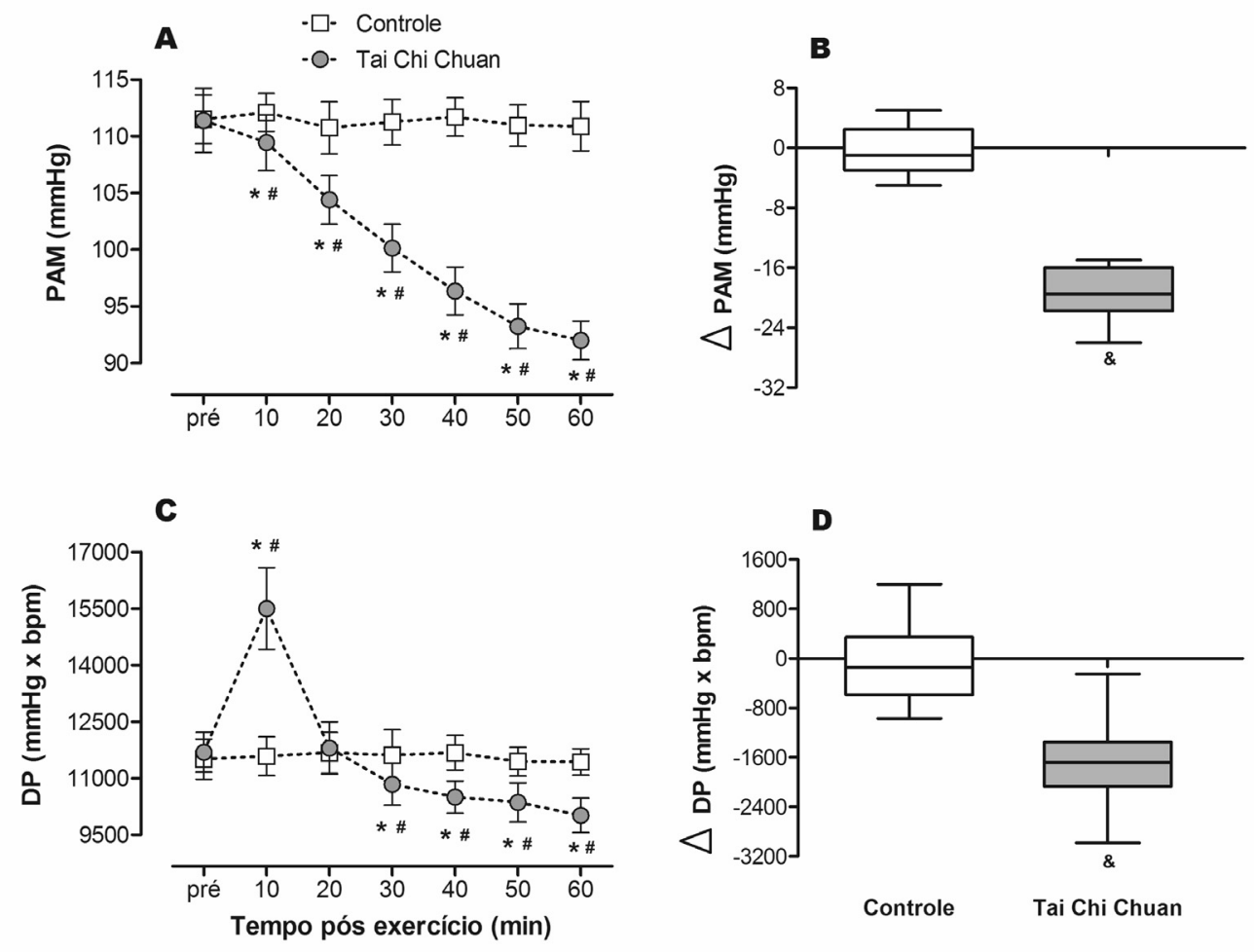

Figura 3. (Painel A e C) Comportamento da pressão arterial média (PAM) e duplo produto (DP) respectivamente, nas sessões controle e após Tai Chi Chuan. (Painel B e D) Diferença entre os valores de PAM e DP respectivamente, após 60 minutos e pré sessões controle e Tai Chi Chuan. *diferente de pré sessão de Tai Chi Chuan; \# diferente do controle; para ANOVA (post-hoc Bonferroni, significância p< $0,05)$. \& diferente do controle; teste T para amostras em pares $(p<0,05)$. Dados apresentados como média \pm desv-pad, $n=20$.

controle da HAS. Adicionalmente, a PAM reduziu significativamente a partir do $10^{\circ}$ minuto após o TCC e o DP apresentou redução significativa a partir do $30^{\circ}$ minuto. Esta resposta do DP ocorreu devido a resposta de redução cronotrópica mais lenta da FC após o exercício. A FC após o TCC permaneceu elevada de maneira significativa até o $30^{\circ}$ minuto, sendo esta resposta esperada devido ao aumento da atividade do sistema nervoso simpático e da liberação de catecolaminas durante o exercício que levam a resposta de FC ficar elevada por certo período de tempo após o exercício. $33,34,35$

Até o presente momento não foi identificado na literatura estudos que analisaram o comportamento da PA e DP após uma sessão de TCC. Um dos raros estudos com este propósito foi o de Chao et al. ${ }^{19} \mathrm{em}$ que analisaram o comportamento da PA após aula de TCC por 30 minutos em 24 mulheres, sendo 9 hipertensas. Nesse estudo os pesquisadores verificaram HPE estatisticamente significativa apenas para a PAS nas mulheres hipertensas, po- rém, diferente do presente estudo, os pesquisadores verificaram HPE para a PAS apenas no $20^{\circ} \mathrm{e}$ $25^{\circ}$ minuto pós-sessão, não havendo HPE para a PAD em nenhum momento após a prática do TCC. Em nosso estudo verificamos redução significativa tanto para a PAS e PAD a partir de $20^{\circ}$ minuto até o $60^{\circ}$ minuto da recuperação, sendo que a magnitude de redução da PA após sessão de TCC em comparação aos valores pré-sessão foram de $\sim 22 \mathrm{mmHg}$ para a PAS e de $\sim 18 \mathrm{mmHg}$ para a PAD.

Um dos fatores a ser considerado e que pode ter determinado a diferença entre os resultados do nosso estudo e o de Chao et al. ${ }^{19}$ são os valores de PAS e PAD pré-exercício, visto que valores mais elevados de PA no momento pré-exercício podem influenciar na magnitude de HPE. ${ }^{16} \mathrm{Em}$ nosso estudo, os idosos apresentavam PAS e PAD pré-exercício elevada $(\sim 152 / 91 \mathrm{mmHg})$, apesar de todos estarem em terapia medicamentosa com anti- hipertensivos, já no estudo de Chao et al. ${ }^{19}$ os valores de PAS e PAD eram de $\sim 121 / 75 \mathrm{mmHg}$. 
Nosso objetivo não foi estudar os mecanismos envolvidos na HPE, no entanto, estudos abordam que a HPE está associada ao aumento de agentes vasodilatadores, como o óxido nítrico, lactato, adenosina e potássio, aumento do tônus parassimpático, reajuste do ponto de controle doa barorreceptores, redução da atividade simpática e da resistência vascular periférica. ${ }^{16,36}$ Além disso, Halliwill et al. ${ }^{18}$ demonstraram recentemente que a HPE é regulada via inibição de receptores neurais chamados de Neurokinin-1 (NK-1), que agem sobre a resposta simpática ativando receptores $\mathrm{H} 1$ e $\mathrm{H} 2$ de histaminas que estão associados a manutenção da vasodilatação.

Analisando as variáveis relacionadas ao exercício físico que mais influenciam a HPE, a literatura demonstra que a intensidade e duração são as principais e exercem grande influência nesta resposta. ${ }^{35,37,38}$ Com relação à intensidade da aula de TCC no presente estudo, não houve interferência dos pesquisadores, pois as aulas de TCC possuem uma característica determinada e um protocolo a ser seguido. $O$ estudo de Lan et al. ${ }^{39}$ analisaram uma típica aula de TCC e identificou que a aula apresentou intensidade moderada, visto que os indivíduos realizavam os exercícios específicos da modalidade em média a $58 \%$ da FC de reserva, $55 \%$ do consumo máximo de oxigênio e concentração de lactato sanguíneo na média de 3,8 $\mathrm{mmol} / \mathrm{l}$ após a prática.

Analisando a relação da duração do exercício e HPE, Forjaz et al. ${ }^{37}$, demonstraram maior HPE após a prática de exercício de maior duração, 10 indivíduos realizaram duas sessões de exercício em bicicleta ergométrica a $50 \%$ do consumo de oxigênio pico, sendo uma sessão com duração de 25 minutos e outra sessão de 45 minutos. Mach et al. ${ }^{40}$ (2005) também demonstraram que exercícios de maior duração promovem maior HPE em pacientes hipertensos. O mesmo não ocorre quando analisado homens jovens normotensos. ${ }^{41}$ Nossos achados corroboram com a literatura, uma vez que foi proposto uma aula de TCC com duração de 60 minutos em idosos hipertensos.

Tem sido proposto recentemente que a equação para o cálculo da PAM logo após o exercício (nos primeiros 2-5 minutos), apresenta um erro considerável devido à perda da relação que se tem do ciclo cardíaco entre a PAS e PAD ( $1 / 3$ e $2 / 3$ respectivamente)..$^{42}$ Logo após esses 5 minutos inici- ais de recuperação do exercício, o cálculo da PAM pela equação tradicional volta a apresentar valores confiáveis. ${ }^{42} \mathrm{Em}$ nosso estudo, não ocorreu esse problema com a interpretação da PAM, pois as medidas de PAS e PAD se iniciaram no $10^{\circ}$ minuto após realização do TCC. E como resultado inédito deste trabalho, observou-se magnitude de redução de PAM após TCC de $\sim 19 \mathrm{mmHg}$ comparado ao momento pré-sessão de TCC. No momento pré-sessão de TCC, a PAM se encontrava elevada, o que demonstra risco para acidente vascular cerebral. ${ }^{43}$

Assim como para a PAM, não foi identificado na literatura dados de DP após sessão de TCC, e o presente estudo demonstrou redução significativa do DP a partir do $30^{\circ}$ minuto que perdurou durante todo o período de monitoramento subsequente. Apesar do DP ser uma análise indireta do consumo de oxigênio pelo miocárdio, apresenta relação direta com a isquemia miocárdica indicando possíveis risco de infarto agudo do miocárdio, sendo que os valores de DP até 30000 é o corte na determinação de uma angina. ${ }^{31,44} \mathrm{O}$ pico de DP encontrado em nosso estudo foi de $\sim 15500$ no $10^{\circ}$ minuto após TCC, demonstrando menor risco de possível angina nos idosos hipertensos.

Embora seja temporário o efeito hipotensor agudo de uma sessão de exercício, esta resposta fisiológica apresenta relevância clínica importante para o controle e tratamento da HAS, pois tem se verificado que o exercício físico diminui cronicamente a PA de repouso, e que essa redução crônica está relacionada ao efeito cumulativo das reduções agudas, o que reforça ainda mais a importância da HPE. ${ }^{36}$ Apesar da característica da aula de TCC ser de movimentos lentos, contínuos e de moderada para baixa intensidade, é um exercício capaz de gerar um estresse no organismo por um tempo suficiente para ativar os mecanismos responsáveis pela $\mathrm{HPE}$, os quais estão relacionados a alterações hemodinâmicas, humorais e neurais.

De acordo com as buscas na literatura, até o presente momento, este é o primeiro trabalho que investigou a HPE, respostas de PAM e DP após monitoramento por 60 minutos do término de uma aula de TCC. Ainda assim encontramos algumas limitações em nosso estudo, como a terapia medicamentosa anti-hipertensiva em que os idosos estavam usando diferentes classes de medicamentos e quantidades, não se sabendo se diferentes drogas po- 
deriam influenciar mais ou menos a HPE, outras limitações foram ainda a falta de randomização da ordem das sessões, o não controle da intensidade da aula de TCC, o baixo número de voluntários idosos na pesquisa e por fim, um tempo curto de monitoramento das variáveis hemodinâmicas após o TCC, o que impossibilita inferir sobre como ficaria o controle pressórico ao longo de um dia.

\section{Conclusão}

Diante dos achados concluímos que uma única sessão de exercício de TCC promove HPE e redução da sobrecarga cardíaca medida pelo menor DP em idosos hipertensos.

\section{Referências}

1. Banco Mundial. Banco Internacional para a reconstrução e o desenvolvimento. Envelhecendo em um Brasil mais veIho: implicações do envelhecimento populacional para o crescimento econômico, a redução da pobreza, as finanças públicas e a prestação de serviço. 2011. [citado em 23 de Dez de 2016]. Disponível em: http:// siteresources.worldbank.org/BRAZILINPOREXTN/ Resources/3817166-1302102548192/Envelhecendo_ Brasil_Sumario_Executivo.pdf

2. Garber CE, Blissmer B, Deschenes MR, Franklin BA, Lamonte $M J$, Lee IM, et al. American college of sports medicine. American College of Sports Medicine position stand. Quantity and quality of exercise for developing and maintaining cardiorespiratory, musculoskeletal, and neuromotor fitness in apparently healthy adults: guidance for prescribing exercise. Med Sci in Sports Exerc. 2011; 43:1334-59.

3. Coin A, Sarti S, Rugggiero E, Giannini S, Pedrazzoni M, Minisola $S$, et al. Prevalence of sarcopenia based on different diagnostic criteria using DEXA and appendicular skeletal muscle mass reference values in an italian population aged 20 to 80. J Am Med Dir Assoc. 2013;14:507-12.

4. Chung JY, Kang HT, Lee DC, Lee HR, Lee YJ. Body composition and its association with cardiometabolic risk factors in the elderly: a focus on sarcopenic obesity. Arch Gerontol Geriatr. 2013; 56:270-78.

5. Hunter GR, McCarthy JP, Bamman M.M. Effects of resistance training on older adults. Sports Med. 2004; 34:32948.

6. Waters DL, Ward AL, Villareal DT. Weight loss in obese adults 65 years and older: A review of the controversy. Exp Gerontol. 2013;48:1054-61.

7. Liu HH, Li JJ. Aging and dyslipidemia: A review of potential mechanisms. Ageing Res Rev. 2015; 19:43-52.

8. Parr EB, Coffey VG, Hawley JA. 'Sarcobesity': A metabolic conundrum. Maturitas.2013;74: 109-13.

9. Poggiogalle E, Migliaccio S, Lenzi A, Donini LM. Treatment of body composition changes in obese and overweight older adults: insight into the phenotype of sarcopenic obesity. Endocrine. 2014;47:699-716.
10. Vincent HK, Raiser SN, Vincent KR. The aging musculoskeletal system and obesity-related considerations with exercise. Ageing Res Rev. 2012; 11:361-73.

11. Sociedade Brasileira de Cardiologia. $7 a$ Diretriz brasileira de hipertensão arterial. Arq Bras Cardiol 2016; 107(3 supl3):1-83.

12. James PA, Oparil S, Carter BL, Pharm D, Cushman WC, Dennison-Himmelfarb C, et al. 2014 evidence-based guideline for the management of high blood pressure in adults: report from the panel members appointed to the Eighth Joint National Committee (JNC 8). JAMA 2014; 311:50720.

13. Leung AA, Nerenberg $K$, Daskalopoulou SS, McBrien K, Zarnke KB, Dasgupta K, et al. Hypertension Canada's 2016 Canadian Hypertension Education Program Guidelines for blood pressure measurement, diagnosis, assessment of risk, prevention, and treatment of hypertension. Can J Cardiol 2016; 32:569-88.

14. Pescatello LS, MacDonald HV, Lamberti L, Jhonson BT. Exercise for hypertension: A prescription update integrating existing recommendations with emerging research. Curr Hypertens Rep 2015; 17:87.

15. Anunciação PG, Polito MD. A Review on post-exercise hypotension in hypertensive individuals. Arq. Bras. Cardiol 2011; 96:e100-e109.

16. Chen $\mathrm{CY}$, Bonham AC. Postexercise hypotension: central mechanisms. Exerc Sport Sci Rev 2010; 38:122-27.

17. Tipton CM. Concerning postexercise hypotension. Exerc Sport Sci Rev 2011; 39:109.

18. Halliwill JR, Buck TM, Lacewell AN, Romero SA. Postexercise hypotension and sustained postexercise vasodilatation: what happens after we exercise? Exp Physiol. 2013; 98:718.

19. Chao CHN, Okano AH, Savir PAH, Alves EA, Elsangedy HM, Cyrino ES, et al. Percepção subjetiva de esforço, resposta afetiva e hipotensão pós-exercício em sessão de Tai Chi Chuan. Motriz 2013; 19:133-40.

20. Lee LY, Lee DT, Woo J. Effect of Tai Chi on state self-steem and health-related quality of life in older chinese residential care home residents. J Clin Nurs 2007; 16:1580-2.

21. Yeh GY, Chan CW, Wayne PM, Conboy L. The impact of tai chi exercise on self-efficacy, social support, and empowerment in heart failure: insights from a qualitative substudy from a randomized controlled trial. Plos One. 2016;11:e0154678.

22. Leung DP, Chan CK, Tsang HW, Tsang WW, Jones AY. Tai chi as an intervention to improve balance and reduce falls in older adults: a systematic and meta-analytical review. Altern Ther Health Med. 2011;17:40-8.

23. Lee LY, Lee DT, Woo J. The psychosocial effect of Tai Chi on nursing home residents. J Clin Nurs 2010; 19:927-38.

24. Song QH, Xu RM, Shen GQ, Zhang QH, Ma M, Zhao XP, et al. Influence of Tai Chi exercise cycle on the senile respiratory and cardiovascular circulatory function. Int J Clin Exp Med. 2014;7:770-4.

25. Taylor-Piliae RE. The effectiveness of Tai Chi exercise in improving aerobic capacity: an updated meta-analysis. Med Sport Sci 2008; 52:40-53.

26. Zheng G, Li S, Huang M, Liu F, Tao J, Chen L. The effect of Tai Chi training on cardiorespiratory fitness in healthy adults: a systematic review and meta-analysis. Plos One 2015; 10:e0117360. 
27. Solloway MR, Taylor SL, Shekelle PG, Miake-Lye IM, Beroes JM, Shanman RM, et al. An evidence map of the effect of Tai Chi on health outcomes. Syst Rev. 2016;5:126.

28. Wang J, Feng B, Yang X, Liu W, Teng F, Li S, et al. Tai Chi for essential hypertension. Evid Based Complement Alternat Med. 2013; 2013:215254.

29. American College of Sports Medicine, Chodzko-Zajko WJ, Proctor DN, Fiatarone Singh MA, Minson CT, Nigg CR, Salem GJ, Skinner JS. American College of Sports Medicine position stand. Exercise and physical activity for older adults. Med Sci Sports Exerc. 2009;41:1510-30.

30. Heyward VH. Avaliação física e prescrição de exercício: técnicas avançadas. 6a ed. Porto Alegre: Artmed; 2013.

31. Powers SK, Howley ET. Fisiologia do exercício: teoria e aplicação ao condicionamento e ao desempenho. $8^{a}$ ed. Barueri: Manole; 2014.

32. McArdle WD, Katch FI, Katch VL. Fisiologia do Exercício: nutrição, energia e desempenho humano. $7^{a}$ ed. Rio de Janeiro: Guanabara Koogan; 2011.

33. Guyton AC, Hall JE. Tratado de fisiologia médica. $12^{\mathrm{a}}$ ed. Rio de Janeiro: Elsevier; 2011.

34. Casonatto J, Polito MD. Hipotensão pós-exercício aeróbio: uma revisão sistemática. Rev Bras Med Esporte. 2009; 15:151-7.

35. Brandão Rondon MUP, Alves MJNN, Braga AMFW, Teixeira OTUN, Barreto ACP, Krieger EM, et al. Postexercise blood pressure reduction in elderly hypertensive patients. J Am Coll of Cardiol. 2002; 39:676-82.

36. Brandão Rondon MUP, Laterza MC, Zamo-Roth FS, Brum PC, Krieger EM. Hipertensão arterial e exercício físico aeróbio. In: Negrão CE, Barreto ACP. Editores. Cardiologia do Exercício: do atleta ao cardiopata. $3^{a}$ ed. Barueri: Manole; 2010. p. 450-69.
37. Forjaz CLM, Santaella DF, Rezende LO, Barreto ACP, Negrão CE. A Duração do exercício determina a magnitude e a duração da hipotensão pós-exercício. Arq Bra Cardiol. 1998; 70:99-104.

38. Jones H, George K, Edwards B, Atkinson G. Is the magnitude of acute post-exercise hypotension mediated by exercise intensity or total work done?. Eur J Appl Physiol. 2007; 102:33-40.

39. Lan C, Chen SY, Lai JS, Wong MK. Heart rate responses and oxygen consumption during Tai Chi Chuan practice. Am J Chi Med. 2001; 29:403-10.

40. Mach C, Foster C, Brice G, Mikat RP, Porcari JP. Effect of exercise duration on postexercise hypotension. J Cardiopulm Rehabil. 2005; 25:366-9.

41. MacDonald JR, MacDougall JD, Hogben CD. The effects of exercise duration on post-exercise hypotension. J Hum Hypertens. 2000; 14:125-9.

42. Sainas G, Milia R, Palazzolo G, Ibba G, Marongiu E, Roberto $S$, et al. Mean blood pressure assessment during postexercise: result from two different methods of calculation. J Sport Sci Med. 2016; 15:424-33.

43. Ladecola C, Davisson RL. Hypertension and cerebrovascular dysfunction. Cell Metab. 2008; 7:476-84.

44. Sociedade Brasileira de Cardiologia. Diretrizes da sociedade brasileira de cardiologia sobre angina estável e infarto agudo do miocárdio sem supradesnível do segmento ST (II edição, 2007) - atualizada 2013/2014. Arq Bra Cardiol. 2014; 102(3supp1). 